\title{
Analyzing Light Scattering Mechanism of Halftoned Prints for Spectral Prediction
}

\author{
Zhonglang Shi ${ }^{1, a}$, Yonghua $\mathrm{Xu}^{1, \mathrm{~b}}$ \\ ${ }^{1}$ Quzhou Fu Teng information technology co.,LTD,Quzhou Electric Power Bureau, Quzhou 324002, \\ China \\ aemail: feijunxu@126.com, ${ }^{\text {bemail: } 164368336 @ q q . c o m}$
}

\begin{abstract}
Keywords: light scattering effect, halftone spectral prediction, probability method, Murray-Davis model
\end{abstract}

\begin{abstract}
Spectral prediction is the key point of halftone reconstruction study, and has always been a meaningful topic of theoretical study. Taking into consideration the translucency of paper and the non-optical contact between the paper and the backing, this paper analyzes the light scattering effect of halftone presswork, deduces the exact expressions of blank area of presswork, and determines the reflectance of the dot part and halftone presswork according to the probability method. Furthermore, a new algorithm for halftone spectral prediction is established. Comparing it with the practical measured spectral reflectivity of the halftone presswork proof by using a spectrophotometer, the accuracy of the model established in this paper is fully verified.
\end{abstract}

\section{Introduction}

Murray[1]first published a simple model to predict output density from input dot area.The Murray-Davis model is by far the most widely used research method of halftone spectral prediction. Since Murray-Davis model ignore the light penetration and scattering in the paper substrate and use of the Murray-Davies model requires making the fundamental assumption that both the substrate and the ink are of uniform color. Although they are conceptually correct it was soon clear that they gave only inaccurate descriptions of experimental results. In 1951, Yule and Nielsen interpreted the discrepancy as the result of light penetration and scattering in the paper substrate[2][3].Yule and Nielsen added the correction coefficient $1 / \mathrm{n}$ based on the reflectivity index of the spectral Murray-Davis model to offset the influence from optical enlargement. However, in practical use, the predicted spectral precision revised by the Yule-Nielsen correction coefficient remains unable to fulfill relevant requirements. Furthermore, solving the coefficient $n$ in terms of the reflectivity index is a difficult problem. Usually, the parameter $\mathrm{n}$ can only be obtained through experimentation. Pearson in 1980 [4], William and Compton in 1989 [5], and ANSI/CGATS 4-1993[6] et al. all pointed out in their respective studies that the Yule-Nielsen correction coefficient $n$ cannot be obtained.

Y.Azuma and M.Inui created a model for predicting the spectra of halftone prints [7].They used a halftone dot structure model to incorporate the effect of light scattering and then estimated the colorimetric characteristics of single dot halftone print. This model assumes that a halftone dot consists of two regions: core and fringe. Rahaman GM Atiqur and Norberg.Ole has a same report about core and fringe of halftone $\operatorname{dot}[8]$. These models share a common "lineage" to the original Murray-Davies model.Arney, Engeldrum, and Zeng [9]; Arney, Wu, and Blehm[10]; and Kruse and Wedin[11] presented first principal model, the models take fundamentally new forms. The first attempts to increase the physical basis of Murray-Davies; this case is extended to color in the second. The last is of a somewhat different spirit than all the previous models. Up to now, ease of measurement and calculation has been important. Now, however, both data collection and model fitting are more difficult, in some cases relying on theoretical rather than empirical parameters. 


\section{Optical analysis of presswork}

In printing, halftone refers to a technique to obtain different shades of a color by controlling size and density of monochrome microscopic dots of ink on the page. The basic geometric analysis of monochrome presswork is illustrated in fig.1, in which the paper-based surface is divided into two parts, with S1 representing the area comprising dots and S2 representing the blank area. For simplification, we assume the ink thickness is uniform.

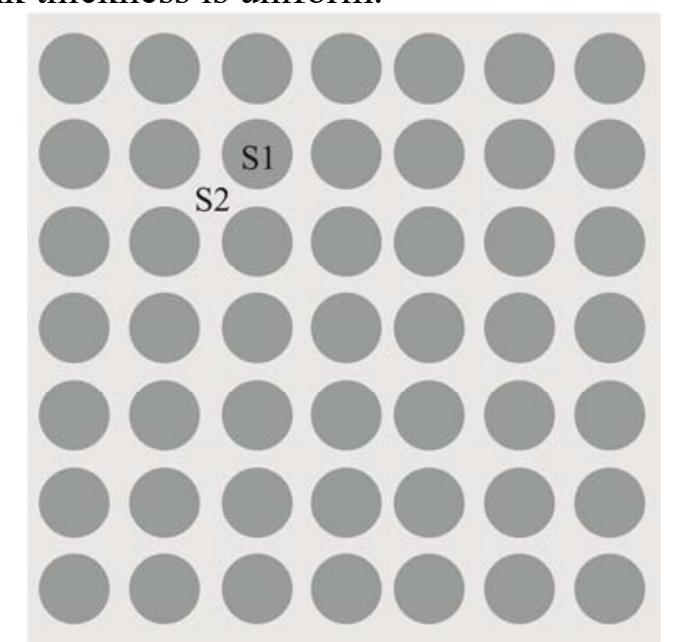

Fig.1:Microscopic image of halftone presswork

The point spread function is a basis tool used to examine the imaging quality of an optical system; it is the value used for calculating the Fraunhofer diffraction integral to the diffraction-restricted imaging system [12].This paper analyzes the light scattering process of microscopic dots as shown in fig. 2. We assume $\mathrm{x}$ indicates any position of the dot part S1, $\mathrm{y}$ indicates any position of the blank paper base $\mathrm{S} 2$, and the photon with $I_{0} d \sigma_{S 1}$ as the light quantity irradiates on dot $\mathrm{x}$, a detector is placed on the $\mathrm{y}$ region to measure the exit light quantity. As such, we assume the photon with $I_{0} d \sigma_{S 2}$ as the light quantity irradiates on dot y, and placed a detector on the $\mathrm{x}$ region to measure the exit light quantity. On the premise of disregarding the influence from ink osmosis and on the condition of consistent illumination, the light from regions S1 to S2 and from S2 to S1 shares the same scattering, according to the light path reversal principle. The scattering light quantity is expressed as

$$
\psi_{S 1-S 2}=\psi_{S 2-S 1}=I_{0} \omega_{S 1 S 2} f(1-f) R_{S 1-S 2}
$$

In the formula, $\psi_{S 1-S 2}$ indicates the scattering light quantity from regions $\mathrm{S} 1$ to $\mathrm{S} 2, \psi_{S 2-S 1}$ represents the scattering light quantity from regions $\mathrm{S} 2$ to $\mathrm{S} 1, I_{0}$ indicates the incident light intensity, $f$ and $(1-f)$ represent the dot and blank area coverage, $R_{S 1-S 2}$ indicates the scattering light reflectance from regions $\mathrm{S} 1$ to $\mathrm{S} 2$ or $\mathrm{S} 2$ to $\mathrm{S} 1, \omega_{S 1 S 2}$ represents the mean value of the integrated point spread function of the light from regions S1 to S2.

The calculation of the quantity $\psi_{S 1-S 2}$ require the knowledge of the point spread function which is usually not available, However if the mean value $\omega_{S 1 S 2}$ of the integrated point spread function of the light from regions $\mathrm{S} 1$ to S2 defined as

$$
\omega_{S 1-S 2}=\frac{1}{f(1-f)} \int_{S 1} \int_{S 2} P(x, y) d \sigma_{S 1} d \sigma_{S 2}
$$

The point spread function $P(x, y)$ indicates the probability of photons entering the dot at position $x$ and exiting from the paper at the position $y$. 


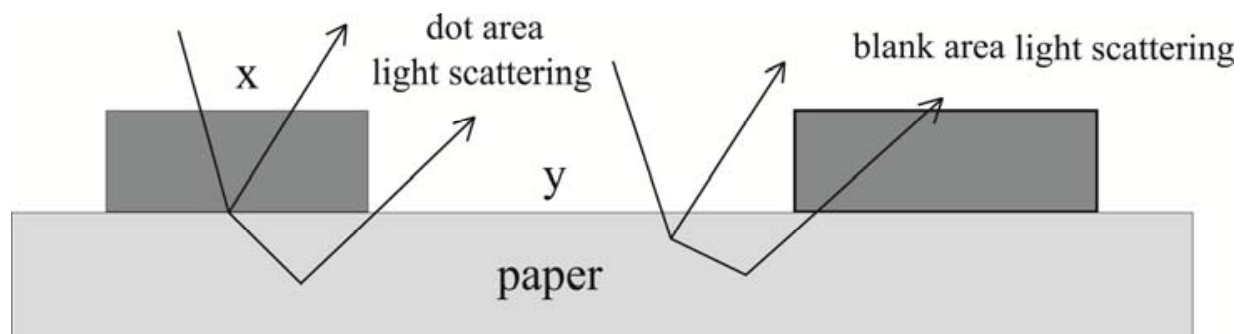

Fig.2: Light scattering on halftone presswork

Due to light irradiating the dot part penetrating into the ink layer, the light entering from the ink layer and exiting from the ink layer (fig.3), the transfer equations of light fluxes traveling between different layers were listed

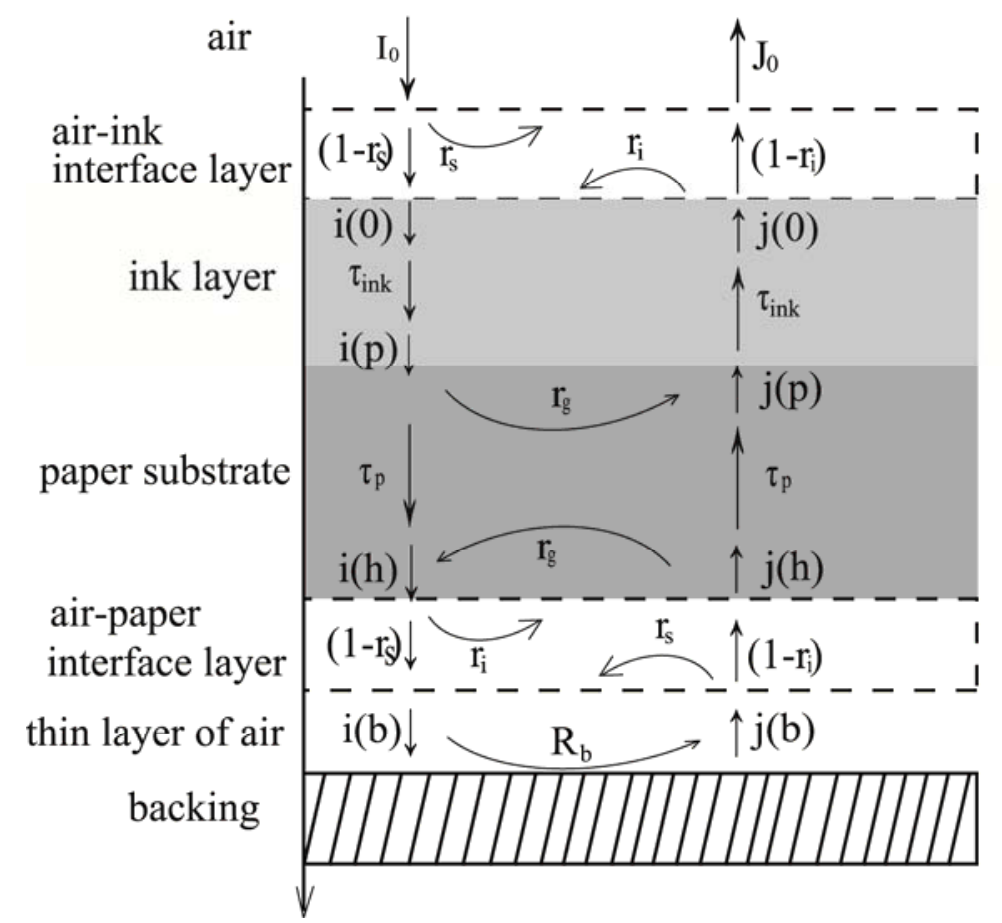

Fig.3: The light entering from the ink layer and exiting from the ink layer

$$
\begin{aligned}
& j(b)=R_{b} i(b) \\
& i(0)=I_{0}\left(1-r_{s}\right)+j(0) r_{i} \\
& j(0)=j(p) \tau_{\text {ink }} \\
& i(p)=i(0) \tau_{\text {ink }} \\
& j(p)=i(p) r_{g}+j(h) \tau_{p} \\
& i(h)=i(p) \tau_{p}+j(h) r_{g} \\
& j(h)=i(h) r_{i}+j(b)\left(1-r_{s}\right) \\
& i(b)=i(h)\left(1-r_{i}\right)+j(b) r_{s} \\
& J_{0}=I_{0} r_{s}+j(0)\left(1-r_{i}\right)
\end{aligned}
$$

As mentioned above,

$$
R_{S 1-S 1}=\frac{J_{0}}{I_{0}}=r_{s}+\frac{\left(1-r_{s}\right)\left(1-r_{i}\right) Z_{1}}{1-r_{i} Z_{1}}
$$

Among, 


$$
Z_{1}=r_{g} \tau_{i n k}^{2}+\frac{\left(1-R_{b} r_{s}\right) r_{i} \tau_{i n k}^{2} \tau_{p}^{2}+\left(1-r_{s}\right)\left(1-r_{i}\right) R_{b} \tau_{i n k}^{2} \tau_{p}^{2}}{\left(1-R_{b} r_{s}\right)\left(1-r_{i} r_{g}\right)-\left(1-r_{s}\right)\left(1-r_{i}\right) R_{b} r_{g}}
$$

In this formulas, $I_{0}$ indicates the incident light intensity of the whole system, $J_{0}$ indicates the emergent light intensity of the whole system, $R_{S 1-S 1}$ indicates the reflectance entering from $\mathrm{S} 1$ and exiting from $\mathrm{S} 1, r_{s}$ represents the specular reflection, $r_{i}$ represents ink internal reflectance, $r_{g}$ represents paper internal reflectance, $R_{p}$ indicates the reflectance of paper-base, $\tau_{i n k}$ represents ink transmittance, $\tau_{p}$ represents paper-based transmittance, $R_{b}$ indicates the reflectance of backing. This parameters $\tau_{p}, r_{s}, r_{i}, r_{g}, \tau_{i n k}$ cannot be obtained by direct method of measurement. To obtain the necessary parameters for our predictive expression a system of equations was established by altering the backing, and measure sample reflectance. Then, we use nlinfit function acquire a non-linear fitting routine. We print the solid using magenta ink on paper-base, and calculate this necessary parameters (fig.4)

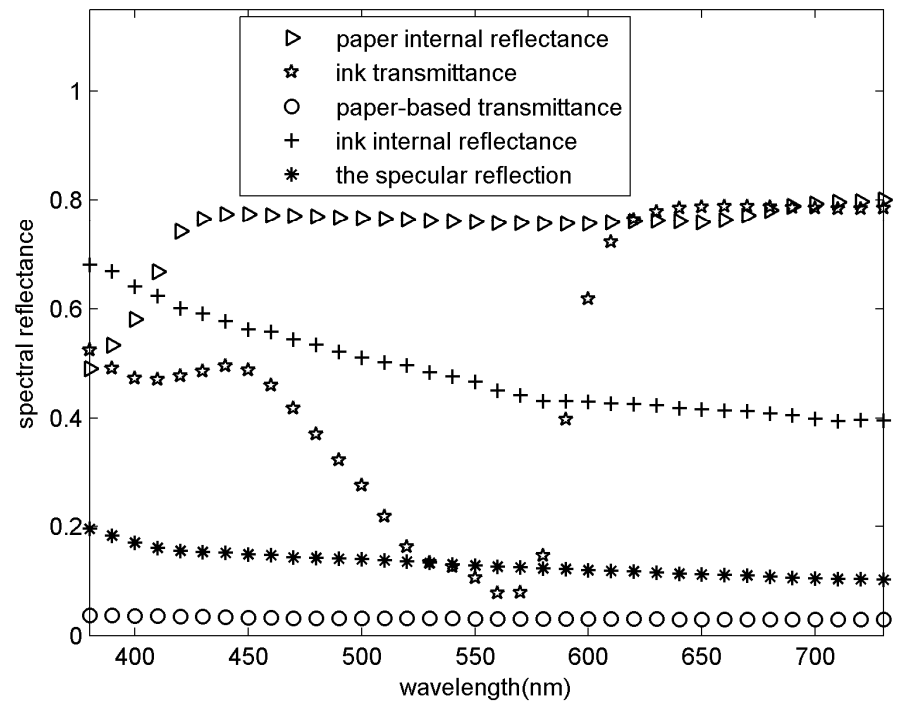

Fig.4 This parameters of the solid print using magenta ink on paper-base

The light entering from the ink layer and exiting from the paper base (or the inverse) shares the same scattering, according to the light path reversal principle, this formulas can all be written as

As such,

$$
R_{S 1-S 2}=R_{S 2-S 1}=r_{s}+\frac{\tau_{i n k}\left(1-r_{s}\right)\left(1-r_{i}\right)\left(r_{g}+Z_{2}\right)}{1-r_{i} \tau_{i n k} r_{g}-r_{i} \tau_{i n k} Z_{2}}
$$

Among,

$$
Z_{2}=\frac{r_{i} \tau_{p}\left(1-r_{s} R_{b}\right)+\tau_{p} R_{b}\left(1-r_{s}\right)\left(1-r_{i}\right)}{1-r_{s} R_{b}-r_{g} r_{i}\left(1-r_{s} R_{b}\right)-R_{b} r_{g}\left(1-r_{s}\right)\left(1-r_{i}\right)}
$$

$R_{S 1-S 2}$ indicates the scattering light reflectance from regions $\mathrm{S} 1$ to $\mathrm{S} 2, R_{S 2-S 1}$ indicates the scattering light reflectance from regions $\mathrm{S} 2$ to $\mathrm{S} 1$.

The light entering and exiting from the paper base,we obtain

$$
R_{S 2-S 2}=\frac{\left(1-r_{s}\right)\left(1-r_{i}\right)\left(r_{g}+Z_{2}\right)}{1-r_{i}\left(r_{g}+Z_{2}\right)}
$$

$R_{S 2-S 2}$ indicates the reflectance from regions $\mathrm{S} 2$ to $\mathrm{S} 2$.

In 1997, Huntsman pioneered the probability model [13], which was then extended by Arney to analyze the scattering of light [14].The present paper analyzes the principle of light scattering by integrating their research methods.

In the case of the reflection and scattering process of light on a microscopic basis, light 
scattering occurs due to the optical penetration of light on presswork under the influence of factors, such as paper base and ink. $P_{S 1-S 1}$ and $P_{S 1-S 2}$ are defined as the probability of light entering from the $\mathrm{S} 1$ region and exiting from the $\mathrm{S} 1$ and $\mathrm{S} 2$ regions; as such, $P_{S 2-S 1}$ and $P_{S 2-S 2}$ are defined as the probability of light entering from the $\mathrm{S} 2$ region and exiting from the $\mathrm{S} 1$ and $\mathrm{S} 2$ regions.

Notably,

$$
\begin{gathered}
P_{S 1-S 1}+P_{S 1-S 2}=1 \\
P_{S 2-S 2}+P_{S 2-S 1}=1
\end{gathered}
$$

Due to light irradiating the dot part penetrating into the ink layer, the transmitted light reflects back by the paper base of ink layer in a process repeated twice, resulting in the following formula:

$$
\psi_{S 1-S 1}=I_{0} f P_{S 1-S 1} R_{S 1-S 1}
$$

The light entering from the ink layer and exiting from the paper base (or the inverse) only passes through the ink layer once, so the formulas can all be written as

$$
\begin{aligned}
& \psi_{S 1-S 2}=I_{0} f P_{S 1-S 2} R_{S 1-S 2} \\
& \psi_{S 2-S 1}=I_{0}(1-f) P_{S 2-S 1} R_{S 2-S 1}
\end{aligned}
$$

The light entering and exiting from the paper base encounters no absorption from the ink layer; therefore

$$
\psi_{S 2-S 2}=I_{0}(1-f) P_{S 2-S 2} R_{S 2-S 2}
$$

The total luminous flux exiting from the blank area (including light entering from dots and exiting from base materials, and those both entering and exiting from base materials) is

$$
\psi_{P}=\psi_{S 1-S 2}+\psi_{S 2-S 2}=I_{0}\left(f R_{S 1-S 2} P_{S 1-S 2}+(1-f) R_{S 2-S 2} P_{S 2-S 2}\right)
$$

The total luminous flux of light in the $\mathrm{S} 1$ region is

$$
\psi_{i}=\psi_{S 1-S 1}+\psi_{S 2-S 1}=I_{0}\left(f R_{S 1-S 1} P_{S 1-S 1}+(1-f) R_{S 2-S 1} P_{S 2-S 1}\right)
$$

Then, the total reflectivity of light exiting from the blank area (including light entering from dots and exiting from base materials, and both those entering and exiting from base materials) can be calculated as

$$
\begin{aligned}
& \rho_{\mathrm{p}}=\frac{\psi_{P}}{(1-f) I_{0}}=\left[P_{\mathrm{S} 1-\mathrm{S} 2} \frac{f}{(1-f)} R_{\mathrm{S} 1-S 2}+P_{S 2-S 2} R_{S 2-S 2}\right] \\
& \rho_{i}=\frac{\psi_{i}}{f I_{0}}=\left[P_{S 1-S 1} R_{S 1-S 1}+P_{S 2-S 1} \frac{(1-f)}{f} R_{S 2-S 1}\right]
\end{aligned}
$$

From eq. (1), eq. (20), and eq. (21), we obtain

$$
P_{S 1-S 2} f=P_{S 2-S 1}(1-f)=\omega_{S 1-S 2} f(1-f)
$$

We then solve for the probability of

$$
P_{S 2-S 2}=1-P_{S 2-S 1}=1-\omega_{S 1-S 2} f
$$

Using the same principle as eq. (28), we achieve

$$
P_{S 1-S 1}=1-P_{S 1-S 2}=1-\omega_{S 1-S 2}(1-f)
$$

eq.(25) and eq. (26) indicate that and are no longer the constants $R_{i}$ and $R_{P}$ in the classic Murray-Davis formula, but have become the precise spectral reflectance considering the light scattering effect and the ink layer has non-uniform thickness. Thus, we know that the precise reflectance of halftone prints is as follows

$$
R_{\text {total }}=\rho_{P}(1-f)+\rho_{i} f
$$

the ultimate expression of halftone spectral prediction model would be

$$
R_{\text {total }}=\left(P_{S 1-S 2} R_{S 1-S 2}+P_{S 1-S 1} R_{S 1-S 1}\right) f+\left(P_{S 2-S 2} R_{S 2-S 2}+P_{S 2-S 1} R_{S 2-S 1}\right)(1-f)
$$

eq. (31) is a very important result in this study.

For convenient application, the mean value $\omega_{S 1 S 2}$ of the integrated point spread function of the light from regions $\mathrm{S} 1$ to S2 may be approximated

$$
\omega_{S 1-S 2}=f^{-\mu},(0 \leq \mu \leq 1)
$$


the suggested value of $\mu$ is 0.53 .

\section{Experimental results}

The measurement instruments used the SpectroEye spectrophotometer,the spectral range of measurement is from $380 \mathrm{~nm}$ to $730 \mathrm{~nm}$ and the interval is $10 \mathrm{~nm}$. The color patches are printed with Heidelberg. The above-deduced point spread and probability model and computational formula of halftone spectral prediction was programmed by MATLAB software, and the spectral reflectances of the pressworks was calculated. The results were compared with actual measured results (fig.5). Due to space constraints, we only adopt the experimental data of top tone dot wedges (30\%,50\%,70\%,90\% cyan,magenta,yellow and black). The expression deduced from this model, which we have verified and tested, also applies to various dot wedge specimen pages of cyan,magenta,yellow and black printing inks,these results can be verified through experiments.
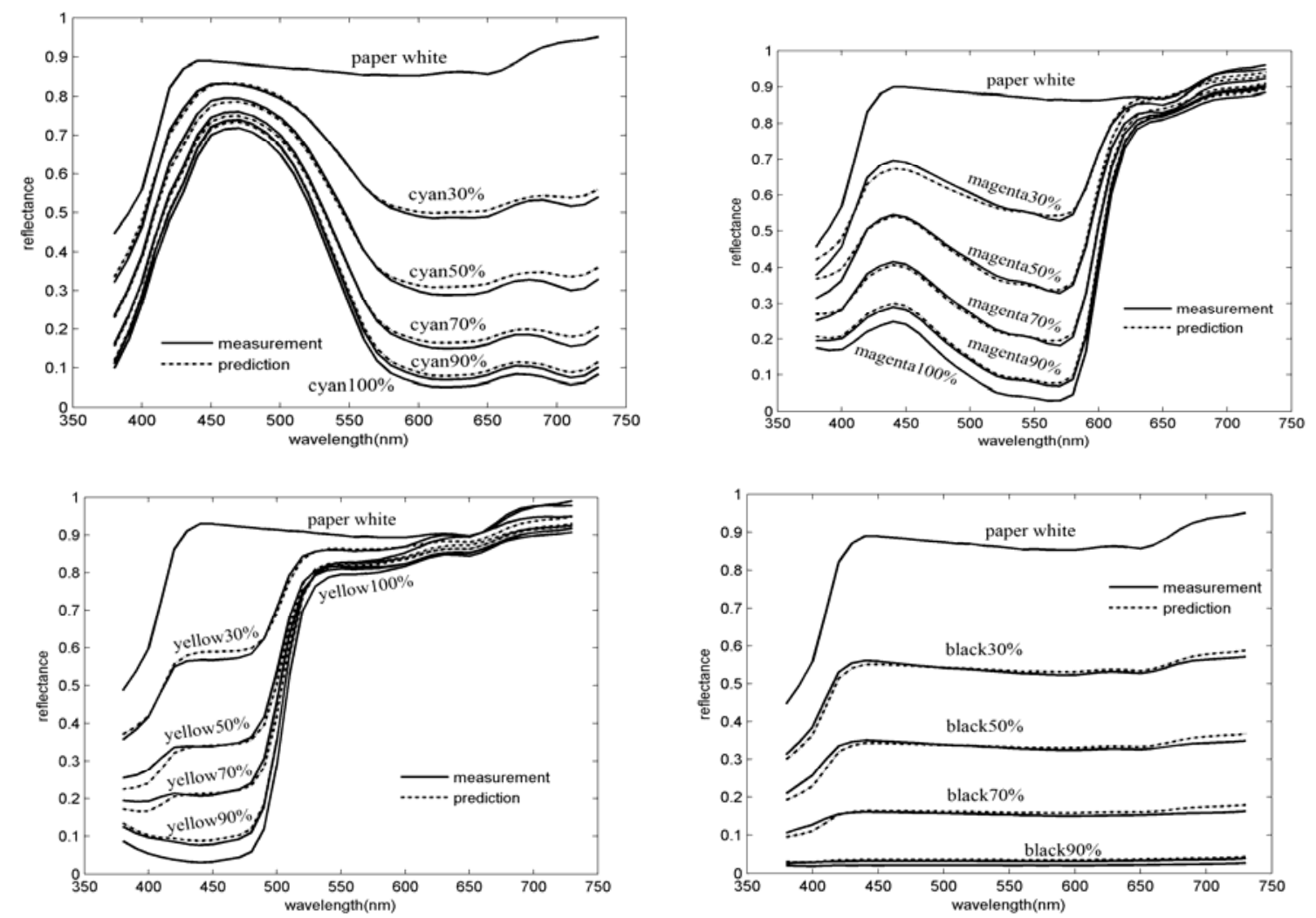

Fig.5:The contrast of the optical spectrum of the pressworks predicted with the actual measured values.

Fig. 5 indicates that by using the model proposed in this paper,the predicted spectral reflectances match well with actual measured values.

\section{Conclusion}

We obtained the formula of the relationship between halftone spectrum and the dot area, the transmittance of the ink layer, the point spread function, and the spectral reflectance of the paper backing. In this study, We acquired favorable results in the spectral reflectance prediction with the formula, demonstrating the precision of the proposed halftone spectral prediction model. This paper only focused on the monochromatic halftone pressworks; as such the penetration effect of the printing ink was not taken into consideration. As for multicolor overprints, the penetration effect of the printing ink requires further study. 


\section{References}

[1]. Murray A, 1936 Monochrome reproduction in photoengraving. J Franklin Inst,221:721-744

[2]. J.A.C.Yule and W.J.Nielsen 1951 The penetration of light into paper and its effect on halftone reproduction Proc TAGA 65-76

[3]. F.R. Ruckdeschel and O.G. Hauser 1978 Yule-Nielsen effect in printing: a physical analysis Appl.Opt.17:3376-3383

[4]. M .Pearson $1980 \mathrm{n}$ value for general conditions Proc TAGA 415-425

[5]. W. P .William 1989 A Practical Approach to N-Value Proc.TAGA142-151

[6]. ANSI:CGATS.4-1993.Graphic technology-Graphic arts reflection densitometry measurements-terminology,equations,image elementsand.procedures Reston.V-A:NPES The Association

for Suppliers of Printing and PublishingTechnol.7

[7]. Y.Azuma and M.Inui 2004 Prediction Color of Halftone Prints using Core-Fringe Model Tokyo Institute of Polytechnics (Japan) 594-597

[8]. Rahaman G M Atiqur, Norberg Ole, Edström Per.2014 Extension of Murray-Davies tone reproduction model by adding edge effect of halftone dots[C],SPIE Proceedings, 90180F-1 90180F-13

[9]. Arney JS, Engeldrum PG, Zeng H. 1995 An expanded Murray-Davies model of tone reproduction in halftone imaging. J Imag Sci Tech 39:502-508

[10]. Arney JS, Wu T, Blehm C. 1998Modeling the Yule-Nielsen effect on color halftones. J Imag Sci Tech 42:335-340

[11]. Kruse B, Wedin M. 1995 A new approach to dot gain modeling. TAGA Proc 47:329 -338

[12]. Jiang.Y.S,Qiu.Z.W,Li.Z, 2004 One-dimensional digital computation and realization on MATLAB of point spread function,Infrared Laser.Eng,33:405-408

[13]. J. Huntsman, 1987 A new model of dot gain and its application to a multilayer color proof,J.Imaging.Sci.Technol.13:136-145

[14]. J.Arney, 1997 A Probability Description of the Yule-Nielsen Effect,I,J.Imaging.Sci.Technol.41:633-636 